\title{
Fibrilhação auricular num ACeS da Região Norte - sua prevalência e proporção de terapêuticas antitrombóticas
}

Diana Oliveira,* Joana Coelho, ${ }^{* *}$ Manuel Magalhães, ${ }^{* *}$ Mariana Oliveira*

\section{RESUMO}

Objetivos: Determinar a prevalência de fibrilhação auricular (FA) no Agrupamento de Centros de Saúde (ACeS) Porto Ocidental e em maio de 2013. Caracterizar a distribuição dos fatores de risco $\mathrm{CHA}_{2} \mathrm{DS}_{2}$ VASc para tromboembolismo, as terapêuticas antitrombóticas e os locais de controlo da hipocoagulação com antagonistas da vitamina K.

Tipo de Estudo: Observacional, transversal e descritivo.

Local: ACeS Porto Ocidental.

População: Utentes inscritos nas Unidades Funcionais do ACeS.

Métodos: Colheita de dados através dos programas SIARS, MIM@UF e SAM. Registo e análise de dados em Microsoft Office Excel 2011 e SPSS 20. O teste qui-quadrado foi utilizado para comparar proporções, aceitando-se um nível de significância de $p<0,05$. Resultados: Identificaram-se 1.628 utentes com FA, 58\% do género feminino; com idade entre 29 e 104 anos sendo a média de $76 \pm 11$. A prevalência global de FA foi de $0,98 \%$ e de $1,77 \%$ nos utentes acima dos 39 anos. Verificou-se que $95 \%$ dos utentes tinha $\mathrm{CHA}_{2} \mathrm{DS}_{2}$ VASc score superior a 0 (excluindo os casos apenas associados ao género feminino). Os fatores de risco mais frequentes foram a hipertensão arterial (75\%), a idade superior a 74 anos (62\%) e o género feminino. A hipocoagulação foi prescrita em $68 \%$ e destes $88 \%$ corresponderam a antagonistas da vitamina $\mathrm{K}$. O controlo laboratorial ocorreu em $74 \%$ dos casos. Conclusões: A prevalência global de FA foi superior à referida num estudo nacional de 2006 (0,5\%). Contudo, a prevalência acima dos 39 anos foi inferior à encontrada num estudo de 2010 , baseado na realização de eletrocardiogramas (2,5\%). O conhecimento da população com FA é importante na otimização de recursos essenciais a uma adequada prestação de cuidados.

PALAVRAS-CHAVE: Fibrilhação auricular; Prevalência; Score $\mathrm{CHA}_{2} \mathrm{DS}_{2}$ VASc; Hipocoagulação.

\section{INTRODUÇÃO}

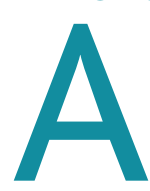

fibrilhação auricular (FA) caracteriza-se por um ritmo irregular, geralmente rápido, constituindo a disritmia sustentada mais frequente e ocorrendo em 1,5 a $2 \%$ da população geral nos países desenvolvidos. ${ }^{1}$ A prevalência de FA aumenta com a idade ${ }^{2-3} \mathrm{e}$, nos Estados Unidos da América, ocorre em $0,1 \%$ das pessoas com menos de 55 anos de idade, 3,8\% das com pelo menos 60 anos e atinge $10 \%$ das com idade igual ou superior a 80 anos. ${ }^{4} \mathrm{O}$ es-

*Médicas internas de Medicina Geral e Familiar, UCSP Foz do Douro - ACeS Porto Ocidental.

**Médicos internos de Medicina Geral e Familiar, USF Porto Douro - ACeS Porto Ocidental. tudo FAMA, realizado em adultos portugueses com idade igual ou superior a 40 anos e publicado em 2010, revelou uma prevalência nacional neste grupo etário de $2,5 \% .{ }^{3}$ Com o aumento da esperança média de vida, estima-se uma duplicação da prevalência de FA na população geral nas próximas décadas..$^{2,5-6}$ A prevalência é frequentemente referida como mais elevada nos indivíduos do género masculino, ${ }^{2}$ todavia os resultados do estudo FAMA não revelaram diferenças estatisticamente significativas entre os dois géneros. Após ajustamento para a idade, verificou-se uma prevalência superior nos homens com idade compreendida entre os 70 e os 79 anos e nas mulheres com pelo menos 80 anos. $^{3}$ 
A FA está intimamente associada a outras doenças cardiovasculares, como insuficiência cardíaca, doença coronária, doença valvular cardíaca, diabetes mellitus e hipertensão arterial (HTA).

O mecanismo de ação pelo qual os fatores de risco cardiovascular predispõem à ocorrência de FA permanece em investigação. Níveis elevados de catecolaminas, stress hemodinâmico ou metabólico, isquemia ou inflamação auricular e ativação da cascata neuro-humoral podem promover o desenvolvimento desta patologia. $^{4}$

A FA aumenta cinco vezes o risco de acidente vascular cerebral (AVC) $)^{1}$ e um em cada cinco destes eventos é atribuído a esta disritmia. Quando associados à FA, os AVCs isquémicos são frequentemente fatais e os doentes que sobrevivem têm maior incapacidade e maior probabilidade de recorrência do que nos restantes casos. $^{2}$

A abordagem de doentes com FA visa reduzir a sintomatologia e as complicações graves desta disritmia, assentando no controlo de ritmo ou frequência e na prevenção de eventos tromboembólicos. A determinação do risco de ocorrência destes eventos é essencial à decisão terapêutica farmacológica, tendo-se baseado durante anos no score CHADS $_{2}$, entretanto substituído pelo score $\mathrm{CHA}_{2} \mathrm{DS}_{2} \mathrm{VASc}$, que se encontra discriminado no Quadro I. ${ }^{1-2}$

De acordo com as recomendações atuais da Sociedade Europeia de Cardiologia, a prevenção farmacológica de eventos tromboembólicos está indicada em doentes com score igual ou superior a 1, excluindo mulheres sem outros fatores de risco. Está recomendada a hipocoagulação oral [International Normalized Ratio (INR) alvo entre 2 e 3], devendo esta ser substituída por antiagregação plaquetária apenas em doentes que recusem a hipocoagulação [associação de ácido acetilsalicílico (AAS) e clopidogrel, reservando-se a monoterapia com AAS para situações de intolerância à terapêutica dupla ou de risco hemorrágico elevado]. Naqueles com score 0 não deverá ser instituída terapêutica anticoagulante ou antiagregante. ${ }^{2}$

Os encargos do Serviço Nacional de Saúde com o tratamento da FA e das suas complicações, a morbimortalidade desta patologia e a previsão do aumento da sua prevalência tornam necessário conhecer a população por ela atingida no ACeS Porto Ocidental, de modo a otimizar os recursos pessoais, técnicos e económicos indispensáveis à adequada prestação de cuidados a estes doentes. Assim, os objetivos principais deste trabalho foram determinar, neste ACeS e em maio de 2013, a prevalência de FA, a proporção dos diferentes riscos tromboembólicos (de acordo com o score $\mathrm{CHA}_{2} \mathrm{DS}_{2}$ VASc - riscos variáveis entre 0 e 9), a proporção de diferentes fatores de risco considerados no score [insuficiência cardíaca, HTA, diabetes mellitus, acidente isquémico transitório (AIT) ou AVC, enfarte agudo do miocárdio (EAM) ou doença arterial periférica, idade compreendida entre 65 e 74 anos e idade igual ou superior a 75 anos], a proporção dos diferentes tipos de terapêutica antitrombótica instituída (nenhuma, antiagregação plaquetária ou hipocoagulação) e a proporção de doentes hipocoagulados com antagonistas da vitamina K controlados a nível laboratorial e hospitalar. Secundariamente, pretendeu-se caracterizar a população com FA quanto ao género e à idade e verificar a existência de diferenças estatisticamente significativas entre a prevalência nos dois géneros.

\section{MÉTODOS}

Com parecer favorável da Comissão de Ética da Administração Regional de Saúde do Norte e colaboração da Presidente do Conselho Clínico do ACeS Porto Ocidental foi efetuado um estudo observacional, transversal e descritivo, durante o período de março a outubro de 2013.

O estudo foi realizado no ACeS Porto Ocidental, incluindo toda a população inscrita nas suas Unidades de Saúde Familiar (USF) e Unidades de Cuidados de Saúde Personalizados (UCSP), em maio de 2013.

Foram definidas as seguintes variáveis de estudo:

- Idade (em anos) dos utentes com FA;

- Unidade Funcional do ACeS, codificada de forma numérica, de modo a garantir o anonimato;

- Insuficiência cardíaca [código K77 da International Classification of Primary Care, Second Edition (ICPC2) presente ou ausente];

- HTA (código K86 ou K87 da ICPC-2 presente ou ausente);

- Diabetes mellitus (código T89 ou T90 da ICPC-2 presente ou ausente);

- AIT e/ ou AVC (código K89 ou K90 da ICPC-2 presente ou ausente); 


\section{QUADRO I. Score CHA $_{2}$ DS $_{2}$ VASc e taxa de AVC}

Fatores de risco para AVC e tromboembolismo na FA não valvular

\begin{tabular}{l|l}
\hline Fatores de risco major & Fatores de risco não major \\
\hline AVC prévio, AIT ou tromboembolismo & Insuficiência cardíaca com disfunção \\
sistémico & sistólica do VE moderada a grave \\
Idade $\geq 75$ anos & (FE VE $\leq 40 \%)$, \\
& hipertensão arterial, diabetes mellitus, \\
& género feminino, 65 a 74 anos de idade, \\
& doença vascular*
\end{tabular}

Abordagem baseada em fatores de risco, expressa num sistema pontuado com o acrónimo $\mathrm{CHA}_{2} \mathrm{DS}_{2}$ VASc

\begin{tabular}{|c|c|c|c|}
\hline \multicolumn{3}{|l|}{ Fatores de risco } & Score \\
\hline \multicolumn{3}{|c|}{ Insuficiência cardíaca congestiva/Disfunção do VE } & 1 \\
\hline \multicolumn{3}{|c|}{ Hipertensão arterial } & 1 \\
\hline \multicolumn{3}{|l|}{ Idade $\geq 75$ anos } & 2 \\
\hline \multicolumn{3}{|l|}{ Diabetes mellitus } & 1 \\
\hline \multicolumn{3}{|c|}{ AVC/AIT/Tromboembolismo } & 2 \\
\hline \multicolumn{3}{|l|}{ Doença vascular* } & 1 \\
\hline \multicolumn{3}{|c|}{ Idade compreendida entre 65 e 74 anos } & 1 \\
\hline \multicolumn{3}{|l|}{ Género feminino } & 1 \\
\hline \multicolumn{3}{|l|}{ Score máximo } & 9 \\
\hline \multicolumn{4}{|c|}{ Taxa ajustada de AVC, de acordo com a pontuação $\mathrm{CHA}_{2} \mathrm{DS}_{2}$ VASc } \\
\hline $\mathrm{CHA}_{2} \mathrm{DS}_{2} \mathrm{VASC}$ & Doentes $(n=7329)$ & \multicolumn{2}{|c|}{ Taxa ajustada de AVC (\%/ano) $\dagger$} \\
\hline 0 & 1 & \multicolumn{2}{|l|}{$0 \%$} \\
\hline 1 & 422 & \multicolumn{2}{|c|}{$1,3 \%$} \\
\hline 2 & 1.230 & \multicolumn{2}{|c|}{$2,2 \%$} \\
\hline 3 & 1.730 & \multicolumn{2}{|c|}{$3,2 \%$} \\
\hline 4 & 1.718 & \multicolumn{2}{|c|}{$4,0 \%$} \\
\hline 5 & 1.159 & \multicolumn{2}{|c|}{$6,7 \%$} \\
\hline 6 & 679 & \multicolumn{2}{|c|}{$9,8 \%$} \\
\hline 7 & 294 & \multicolumn{2}{|c|}{$9,6 \%$} \\
\hline 8 & 82 & \multicolumn{2}{|c|}{$6,7 \%$} \\
\hline 9 & 14 & \multicolumn{2}{|c|}{$15,2 \%$} \\
\hline
\end{tabular}

* Enfarte do miocárdio prévio, doença arterial periférica, placa aórtica. As taxas de AVC em coortes atuais podem variar destas estimativas.

† Baseado em Lip et al. ${ }^{7}$

Legenda: AIT = acidente isquémico transitório; $A V C$ = acidente vascular cerebral; FA = fibrilhação auricular; $\mathrm{FE}=$ fração de ejeção; $\mathrm{VE}=$ ventrículo esquerdo
- $\mathrm{CHA}_{2} \mathrm{DS}_{2} \mathrm{VASc}$ (pontuação do score, de 0 a 9 );

- Tratamento antitrombótico [nenhum, antiagregação plaquetária ou hipocoagulação (acenocumarol, varfarina ou outros)];

- Local de controlo de hipocoagulação (laboratório ou hospital).

Foi obtido o número de utentes inscritos nas USF e UCSP do ACeS através do programa Sistema de Informação da Administração Regional de Saúde (SIARS) e foram identificados os utentes com FA mediante o programa Módulo de Informação e Monitorização das Unidades Funcionais (MIM@UF)-identificação restrita aos utentes com codificação K78 da ICPC-2 no processo clínico Sistema de Apoio ao Médico (SAM). Os dados relativos ao género e à idade dos utentes foram colhidos do programa MIM@UF e a restante informação necessária à realização do trabalho adveio da consulta individual dos processos clínicos SAM dos utentes identificados com FA.

A análise estatística foi realizada através dos programas Microsoft Office Excel 2011 e SPSS versão 20 . O teste de quiquadrado foi usado para avaliar a associação entre variáveis categóricas. Na análise estatística foi considerado significado estatístico quando $p<0,05$.
- EAM e/ou doença arterial periférica (código K75 ou K92 da ICPC-2 presente ou ausente);

- Género (masculino ou feminino);

\section{RESULTADOS}

Em maio de 2013 foram identificados 159.702 utentes inscritos nas USF e UCSP do ACeS Porto Ocidental, 
1.628 dos quais com diagnóstico de FA. Foram excluídos do estudo 66 utentes com diagnóstico de FA por: cardioversão prévia resultando em ritmo sinusal (22), o número de utente obtido através do programa MIM@UF não ser aceite no SAM (9), transferência de Unidade Funcional (9), aparente erro de codificação diagnóstica (2) e falecimento (24). Foram, assim, incluídos no estudo 1.562 indivíduos, tendo sido determinada uma prevalência de FA de $0,98 \%$ (IC $95 \%=0,93$ - 1,03). Em utentes com pelo menos 40 anos de idade foi determinada uma prevalência de $1,77 \%$ (IC $95 \%=$ $1,68-1,86)$.

Cinquenta e oito por cento dos utentes incluídos no estudo são do género feminino. A idade dos utentes está compreendida entre os 29 e os 104 anos (média de $76 \pm 11$ anos).

Foi determinada uma prevalência de FA de $1,03 \%$ no género feminino (IC $95 \%=0,97-1,10 \%$ ) e de $0,91 \%$ no género masculino (IC $95 \%=0,84-0,98 \%)(p=0,012)$.

O Quadro II revela a proporção dos diferentes riscos tromboembólicos (de acordo com o score $\mathrm{CHA}_{2} \mathrm{DS}_{2} \mathrm{VASc}$ ), verificando-se que $97 \%$ dos utentes com FA tinha score igual ou superior a 1 , tendo a maioria score de 3 e 4 . Excluindo as mulheres sem outros fatores de risco, 95\% dos utentes com FA tinha indicação para hipocoagulação.

O Quadro III revela a proporção dos diferentes fatores de risco considerados no score $\mathrm{CHA}_{2} \mathrm{DS}_{2}$ VASc, constatando-se predomínio de HTA (75\%), idade igual ou superior a 75 anos (62\%) e género feminino (58\%).

A maioria dos utentes com FA encontrava-se hipocoagulada (68\%), sobretudo com acenocumarol ou varfarina (Quadro IV). Entre os doentes com FA, 16\% estavam medicados com antiagregante plaquetário, $3 \%$ não realizavam terapêutica antitrombótica e em 13\% dos casos a terapêutica era desconhecida.

O local de controlo dos doentes hipoacoagulados com antagonistas da vitamina $\mathrm{K}$ foi em $74 \%$ dos casos um laboratório de análises clínicas. Em $12 \%$ dos casos, os doentes realizavam o controlo de INR em ambiente hospitalar, desconhecendo-se o local de vigilância em $14 \%$ destes utentes.

\section{DISCUSSÃO}

A prevalência de FA determinada em adultos com pelo menos 40 anos de idade $(1,77 \%)$ foi inferior à obti-

\begin{tabular}{|c|c|}
\hline QUADRO II. Proporção dos diferentes riscos \\
tromboembólicos $(\mathbf{n}=1.562)$ \\
Score CHA ${ }_{2}$ SS $_{2}$ VASc & Proporção de utentes com FA \\
\cline { 2 - 2 } 0 & $\mathbf{n}(\%)$ \\
\hline 1 & $41(2,6)$ \\
2 & $111(7,1)$ \\
3 & $198(12,7)$ \\
4 & $393(25,2)$ \\
5 & $437(28,0)$ \\
6 & $216(13,8)$ \\
7 & $117(7,5)$ \\
8 & $39(2,5)$ \\
9 & $8(0,5)$ \\
\end{tabular}

QUADRO III. Proporção dos diferentes fatores de risco considerados no score $\mathrm{CHA}_{2} \mathrm{DS}_{2}$ VASc $(n=1.562)$

\begin{tabular}{l|c}
\multirow{2}{*}{ Fatores de risco } & Proporção de utentes com FA \\
\cline { 2 - 2 } & $\mathbf{n}(\%)$ \\
\hline IC & $444(28)$ \\
HTA & $1.178(75)$ \\
DM & $416(27)$ \\
AVC/AIT & $224(14)$ \\
EAM/DAP & $179(11)$ \\
65-74 Anos & $353(23)$ \\
75 Anos & $976(62)$ \\
Género feminino & $906(58)$
\end{tabular}

Legenda: $\mathrm{AIT}=$ acidente isquémico transitório; $\mathrm{AVC}=$ acidente vascular cerebral; DAP = doença arterial periférica; DM = diabetes mellitus; EAM = enfarte agudo do miocárdio; HTA = hipertensão arterial; IC = insuficiência cardíaca.

QUADRO IV. Proporção dos diferentes tipos de terapêutica anticoagulante $(n=1.062)$

Tipo de terapêutica anticoagulante $\mathrm{n}(\%)$

Acenocumarol $541(51)$

Varfarina 393 (37)

Dabigatrano

$117(11)$

Enoxaparina

$1(0,1)$

Desconhecido

10 (1) 
da pelo estudo FAMA (2,5\%). No ACeS Porto Ocidental, a prevalência de FA de $0,98 \%$ poderá ser inferior à real. Possíveis justificações são a discrepância que se tem verificado entre o número de utentes inscritos nas USF e UCSP obtido por diferentes programas informáticos [Sistema Informático de Unidades de Saúde (SINUS), SIARS e MIM@UF], assim como o facto de a totalidade dos inscritos no ACeS Porto Ocidental ter sido obtida através do SIARS e os utentes com FA terem sido identificados através do MIM@UF, com inclusão daqueles registados no SAM com o código K78 da ICPC-2 e excluídos os submetidos a cardioversão sem evidência de manterem FA. Poderá existir também um eventual subdiagnóstico de FA, como apontado no estudo FAMA, no qual a população selecionada foi submetida a eletrocardiograma, com consequente identificação de casos sem diagnóstico prévio e ainda uma eventual subcodificação de casos de FA diagnosticados.

No entanto, a prevalência de FA determinada na população inscrita no ACeS Porto Ocidental foi superior à referida num estudo português publicado em 2006 na Revista Portuguesa de Clínica Geral, realizado por Médicos Sentinela dos Cuidados de Saúde Primários $(0,5 \%){ }^{8}$

Contrariamente ao estudo FAMA, o presente estudo revelou diferenças estatisticamente significativas entre a prevalência de FA nos dois géneros. Embora a prevalência seja referida frequentemente como mais elevada no género masculino, este estudo revelou o oposto, o que poderá ser consequência da maior utilização dos cuidados de saúde primários por utentes do género feminino, já que dados divulgados pelo Boletim Estatístico da Administração Regional de Saúde de Lisboa e Vale do Tejo apontam para um predomínio da procura de cuidados de saúde, a partir dos 15 anos, pelo género feminino. ${ }^{9}$

De acordo com o score $\mathrm{CHA}_{2} \mathrm{DS}_{2} \mathrm{VASc}, 95 \%$ dos doentes com FA do ACeS tinha indicação para hipocoagulação. Porém, apenas $68 \%$ dos mesmos se encontrava hipocoagulado. Dado a última publicação da Sociedade Europeia de Cardiologia relativa à FA recomendar que a avaliação do risco hemorrágico não conduz, por si só, à exclusão do tratamento hipocoagulante, mas à correção de fatores de risco hemorrágico, torna-se importante rever o motivo pelo qual $32 \%$ dos utentes com FA não estava a fazer este tratamento. São apontadas di- versas razões para a subutilização de hipocoagulação, ${ }^{10-14}$ entre as quais, receio de hemorragias ou experiências prévias de ocorrência de eventos hemorrágicos pela parte dos médicos e baixos níveis de início de tratamento, existência de uma margem terapêutica estreita que obriga a monitorização do INR e baixa adesão do doente. Em relação ao presente estudo, devem ser consideradas uma eventual justificação clínica para a não instituição de hipocoagulação ou uma escassez na difusão das alterações propostas pela Sociedade Europeia de Cardiologia, em 2012. Além disso, em alguns casos, o score poderá estar sobrestimado pelo facto dos autores do presente estudo terem incluído como fator de risco $\mathrm{CHA}_{2} \mathrm{DS}_{2} \mathrm{VASc}$ a presença de codificação K77 da ICPC-2 (alusiva à insuficiência cardíaca), apesar de o score considerar que esta entidade clínica apenas constitui fator de risco perante disfunção sistólica ventricular esquerda moderada a grave. Em alguns casos, o $s c o-$ re poderá estar subestimado, dado o estudo não ter incluído todos os fatores de risco $\mathrm{CHA}_{2} \mathrm{DS}_{2} \mathrm{VASc}$, mas apenas aqueles passíveis de estarem acessíveis no SAM. Uma das maiores limitações deste estudo foi o subregisto nos processos clínicos.

Oitenta e oito por cento dos doentes hipocoagulados por FA estavam medicados com fármacos antagonistas da vitamina $K$, que implicam o controlo de INR, realizado a nível laboratorial em $74 \%$ dos casos.

O presente trabalho incluiu não só os doentes com FA, mas também aqueles com flutter auricular, dado estarem codificados com K78 da ICPC-2. Esta última arritmia muitas vezes padece ou alterna com a FA e apresenta um risco semelhante de AVC e idêntica terapêutica profilática. ${ }^{3}$

Para além das referidas limitações do trabalho, acrescenta-se o facto de o programa SIARS não ter disponibilizado o número de utentes inscritos em maio de 2013 numa das Unidades estudadas, pelo que foi utilizado o número de utentes alusivo a fevereiro do mesmo ano.

Os dados utilizados na realização deste trabalho foram obtidos através de programas informáticos. Para fins de investigação em Cuidados de Saúde Primários é necessário que os dados administrativos que são disponibilizados aos profissionais de saúde sejam fiáveis, devendo ser dada maior atenção à necessidade de melhoria da qualidade dos mesmos. No sentido de assegurar um máximo de fiabilidade das estatísticas inferi- 
das, esta deverá ser investigada noutros estudos.

Não obstante as limitações, este estudo permite caracterizar a população de utentes com FA no ACeS Porto Ocidental, o que ainda não tinha sido efetuado. Sendo a FA a disritmia mais frequente e estimando-se um aumento da sua prevalência na população geral nas próximas décadas, estudos que permitam conhecer as populações por ela atingidas revelam-se importantes $\mathrm{e}$ poderão auxiliar na otimização de recursos fundamentais a uma prestação de cuidados adequada.

\section{REFERÊNCIAS BIBLIOGRÁFICAS}

1. Camm AJ, Kirchhof P, Lip GY, Schotten U, Savelieva I, Ernst S, et al. Guidelines for the management of atrial fibrillation: the Task Force for the Management of Atrial Fibrillation of the European Society of Cardiology. Eur Heart J. 2010;31(19):2369-429.

2. Camm AJ, Lip GY, De Caterina R, Savelieva I, Atar D, Hohnloser SH, et al. 2012 focused update of the ESC Guidelines for the management of atrial fibrillation: an update of the 2010 ESC Guidelines for the management of atrial fibrillation. Developed with the special contribution of the European Heart Rhythm Association. Eur Heart J. 2012;33(21):2719-47.

3. Bonhorst D, Mendes M, Adragão P, Sousa J, Primo J, Leiria E, et al. Prevalência de fibrilhação auricular na população portuguesa com 40 anos ou mais: estudo FAMA [Prevalence of atrial fibrillation in the Portuguese population aged 40 and over: the FAMA study]. Rev Port Cardiol. 2010;29(3):331-50. Portuguese

4. Rosenthal L. Atrial fibrillation [homepage]. Medscape; 2014 [revised 2013 Mar 5; cited 2014 Jan 8]. Available from: http://emedicine.medscape.com/article/151066-overview

5. Stewart S, Hart C, Hole D, McMurray J. Population prevalence, incidence, and predictors of atrial fibrillation in the Renfrew/Paisley study. Heart. 2001;86(5):516-21.

6. Go AS, Hylek EM, Phillips KA, Chang Y, Henault LE, Selby JV, et al. Prevalence of diagnosed atrial fibrillation in adults: national implications for rhythm management and stroke prevention: the AnTicoagulation and Risk Factors in Atrial Fibrillation (ATRIA) Study. JAMA. 2001;285 (18):2370-5.

7. Lip GY, Frison L, Halperin JL, Lane DA. Identifying patients at high risk for stroke despite anticoagulation: a comparison of contemporary stro- ke risk stratification schemes in an anticoagulated atrial fibrillation cohort. Stroke. 2010;41(12):2731-8.

8. Ascenção P. Fibrilhação auricular e prevenção do tromboembolismo: estudo numa população de utentes de Centros de Saúde. Rev Port Clin Geral 2006;22(1):13-24. Portuguese

9. Administração Regional de Saúde de Lisboa e Vale do Tejo. Boletim estatístico: Janeiro-Setembro 2013, Cuidados de Saúde Primários (CSP) [homepage]. Lisboa: ARSLVT [cited 2014 Jun 5]. Available from: http://www.arslvt.min-saude.pt/nucleoestudosplaneamento/Documents/Boletins\%20Estat\%C3\%ADsticos/Boletim\%20CSP_3\%C2\%BA Trimestre\%202013_10_2013_vf.pdf. Portuguese

10. Ogilvie IM, Newton N, Welner SA, Cowell W, Lip GY. Underuse of oral anticoagulants in atrial fibrillation: a systematic review. Am J Med. 2010;123(7):638-45.

11. Gattellari M, Worthington J, Zwar N, Middleton S. Barriers to the use of anticoagulation for nonvalvular atrial fibrillation: a representative survey of Australian family physicians. Stroke. 2008;39(1):227-30.

12. Anderson N, Fuller R, Dudley N. 'Rules of thumb' or reflective practice? Understanding senior physicians' decision-making about antithrombotic usage in atrial fibrillation. QJM. 2007;100(5):263-9.

13. Gandolfo C, Balestrino M, Burrone A, Del Sette M, Finocchi C. Stroke due to atrial fibrillation and the attitude to prescribing anticoagulant prevention in Italy: a prospective study of a consecutive stroke population admitted to a comprehensive stroke unit. J Neurol. 2008;255(6):796-802.

14. Reynolds MR, Shah J, Essebag V, Olshansky B, Friedman PA, Hadjis T, et al. Patterns and predictors of warfarin use in patients with new-onset atrial fibrillation from the FRACTAL Registry. Am J Cardiol. 2006;97(4):538-43.

\section{CONFLITOS DE INTERESSE}

Os autores declaram não ter conflito de interesses.

\section{ENDEREÇO PARA CORRESPONDÊNCIA}

Mariana Filipa Fraga de Oliveira

R. Professor Egas Moniz, n 1475

4510-119 Jovim-Gondomar

E-mail: mariana.ffo@gmail.com

Recebido em 21-01-2014

Aceite para publicação em 06-10-2014 


\section{ABSTRACT \\ PREVALENCE OF ATRIAL FIBRILLATION AND ANTITHROMBOTIC THERAPY IN A GROUP OF PRIMARY HEALTH CARE CLINICS IN THE NORTH OF PORTUGAL}

Objectives: To determine the prevalence of atrial fibrillation among patients in primary health care clinics in West Porto in May 2013, to assess the distribution of $\mathrm{CHA}_{2} \mathrm{DS}_{2} \mathrm{VASC}$ risk factors for thromboembolism, to study antithrombotic therapy and the sites where anticoagulation therapy is managed.

Type of Study: Cross-sectional study.

Setting: Primary Health Care Centres of West Porto.

Population: Patients registered in the health units of West Porto.

Methods: Data were collected from the electronic medical records using SIARS, MIM@UF and SAM software. The chi-squared statistic was used for comparison of proportions, with statistical significance set at the $p<0.05$ level.

Results: The authors identified 1628 people with atrial fibrillation. Of these $58 \%$ were female. They ranged in age from 29 to 104 years with a mean age of $76 \pm 11$ years. The prevalence of atrial fibrillation was $0.98 \%$, with a prevalence of $1.77 \%$ in patients over 39 years. The $\mathrm{CHA}_{2} \mathrm{DS}_{2}$ VASc score was greater than zero in $95 \%$ of patients, excluding cases where female gender was the only risk factor. The main risk factors were arterial hypertension (75\%), age greater than 74 years (62\%) and female gender. Anticoagulation therapy was prescribed to $68 \%$ of patients with $88 \%$ receiving vitamin $\mathrm{K}$ antagonists. Follow-up of anticoagulation was performed in clinical laboratories in $74 \%$ of cases.

Conclusions: The prevalence of atrial fibrillation in this population was greater than that reported in a national study in 2006 $(0.5 \%)$. However the prevalence in patients over 39 years of age was less than that found in a study conducted in 2010, using electrocardiographic diagnosis (2.5\%). Identification of patients with atrial fibrillation is an important task in primary health care.

Keywords: Atrial Fibrillation; Prevalence; $\mathrm{CHA}_{2} \mathrm{DS}_{2}$ VASc Score; Anticoagulation. 